\title{
The utility of upper urinary tract urine cytology before and after application of the Paris system
}

\author{
Caroline Talsma Simon MD ${ }^{1}$ | Stephanie L. Skala MD ${ }^{1}$ | Martin J. Magers MD ${ }^{2}$ | \\ Alon Weizer $\mathrm{MD}^{3}$ | Samuel D. Kaffenberger $\mathrm{MD}^{3}$ | Arul M. Chinnaiyan $\mathrm{MD}^{1}$ | \\ Daniel E. Spratt MD ${ }^{3}$ | Jeffrey Montgomery MD | Rohit Mehra MD ${ }^{1}$ | Madelyn Lew MD $^{1}$ (1)
}

${ }^{1}$ Department of Pathology, University of Michigan Health System, Ann Arbor, Michigan

${ }^{2}$ Department of Pathology, Indiana University, Indianapolis, Indiana

${ }^{3}$ Department of Urology, University of Michigan Health System, Ann Arbor, Michigan

Correspondence

Madelyn Lew, MD, Department of Pathology,

University of Michigan Health System, 1500

E. Medical Center Drive, Ann Arbor, MI

48109-5054.

Email: lewm@med.umich.edu
Background: The Paris System (TPS) introduced diagnostic criteria for urine cytology to improve reproducibility among pathologists. Thus far, most cytology studies have investigated application of TPS on lower urinary tract specimens. Also, it is unclear which cytologic features are most predictive of malignancy, particularly in the upper urinary tract. We evaluate concordance rates of preoperative upper urinary tract cytology specimens before and after application of TPS criteria with surgical resections and assess cytologic features associated with malignancy.

Design: 54 resections with high- and low-grade urothelial carcinoma (HGUC, LGUC) from 2000-2016 with available preoperative cytology $(n=61)$ were identified. Cytology was re-reviewed to evaluate cytologic features and provide diagnoses before and after TPS implementation.

Results: The most common cytologic features associated with HGUC were N:C ratios $\geq 0.7$ (88\%), hyperchromasia (83\%), coarse chromatin distribution (67\%), and nuclear pleomorphism in cell clusters (65\%). Application of TPS criteria resulted in 10 diagnostic downgrades and 6 diagnostic upgrades. After TPS criteria were applied, the sensitivity of a positive diagnosis decreased from $29 \%$ to $19 \%$. The morphologic feature most consistently associated with a downgrade from positive to suspicious was a lack of marked nuclear contour irregularities in atypical urothelial cells.

Conclusion: Using strict TPS criteria in upper urinary tract cytology specimens may decrease the frequency of positive diagnoses with a concurrent increase in suspicious diagnoses. These findings may indicate that different morphologic features, particularly markedly irregular nuclear contours, may have different predictive values for HGUC in upper urinary tract cytology specimens compared with those from the lower urinary tract.

\section{KEYWORDS}

Paris system, upper urinary tract, urine, urine cytology

\section{1 | INTRODUCTION}

Primary cancers of the urinary tract represent the ninth most common site of cancer worldwide. ${ }^{1}$ Upper tract urothelial cancers (UTUC) are relatively rare, accounting for approximately $5 \%-10 \%$ of all urothelial cell carcinomas and $5 \%-7 \%$ of renal neoplasms. ${ }^{2}$ When compared with urothelial carcinomas of the lower urinary tract, UTUC are less common, but also confer a worse prognosis, leading to an estimated 15,930 deaths in the United States in $2018 .^{3}$ Additionally, the propensity of urothelial carcinoma for recurrence and multifocality results in increased surveillance and monitoring to guide patient management. As such, urothelial carcinoma results in the highest lifetime financial cost for a patient of any cancer. ${ }^{4}$

Although voided urine cytology (VUC) has been shown to be a convenient method of diagnosing high-grade urothelial carcinoma (HGUC) in the lower urinary tract, diagnosing upper urinary tract HGUCs by VUC is limited by poor cellular preservation and mechanical distortion. ${ }^{5,6}$ Additionally, the increased technical difficulty in visualizing and accessing potential tumors in the upper urinary tract in comparison to those arising in the lower urinary tract also makes 
acquisition of diagnostic material by biopsy more difficult. ${ }^{7}$ These factors highlight the utility of upper urinary tract washing cytology specimens, as there is a wider area of urothelium evaluated during an upper tract washing compared with a focused biopsy. Furthermore, cytologic atypia in upper urinary tract HGUC are more prominently featured in upper tract cytology washes in comparison to VUC due to better cellular preservation.

However, the utility of urine cytology has historically been limited by the variable usage of indeterminate categories such as "atypical" and "suspicious," which in turn leads to confusion as to what management options should be pursued by the clinicians. As such, there have previously been several attempts to standardize diagnoses in urine cytology by various classification schema. ${ }^{8}$ Currently, the most formal and widely utilized classification system has been the recently published Paris System for Urine Cytology.

The Paris System (TPS) introduced diagnostic morphologic criteria for urine cytology to improve reproducibility among pathologists and potentially help establish management guidelines. Thus far, many cytology studies have focused on the application of TPS on urine specimens from the lower urinary tract. Additionally, there is still uncertainty as to which cytologic features are most predictive of malignancy, particularly in upper tract urine cytology. This study evaluates the concordance rates of preoperative upper tract urine cytology specimens before and after application of TPS criteria with surgical resection specimens as well as cytologic features most predictive of malignancy.

\section{2 | MATERIALS AND METHODS}

With Institutional Review Board approval, 114 patients with a diagnosis of upper urinary tract urothelial carcinoma who had undergone radical nephroureterectomy or ureterectomy between 2000 and 2016 were retrospectively identified from the surgical pathology archive at Michigan Medicine. Of these, 59 patients had both surgical pathology and pre-operative urine cytology specimens (ThinPrep) available for review for a total of 61 resections and 111 cytology specimens. Patients with non-diagnostic cytologic specimens, no residual disease on surgical resection, and/or only voided or urinary bladder washing urine cytology (no upper tract urine cytology) were excluded from the cohort. Also, cases in which the pre-operative urine cytology specimens were collected only from the contralateral side of the ultimate surgical resection or were collected over a year from the date of surgical resection were excluded from the cohort. This left a total of 53 patients with 54 resections and 72 cytology specimens. In this cohort, there were 7 patients who had more than 1 upper urinary tract cytology specimen collected concurrently from the same site. A total of 4 patients had 2 concurrent samples, 2 patients had 3 concurrent samples, and 1 patient had 4 concurrent samples. In these instances, the urine cytology with the most severe diagnosis for each of these patients was used for analysis. These final parameters left a total of 53 patients with 54 resections and 61 urine cytologic specimens for evaluation.

The cytology materials were reviewed by a cytopathologist (M.L.) who was blinded to patient demographics and clinicopathologic data.
The cytopathologist evaluated each cytology specimen and provided diagnoses before and after application of diagnostic criteria put forth by TPS. In the initial evaluation of urine specimens before application of TPS criteria, the diagnostic categories of "no malignant cells identified," "atypical urothelial cells," "suspicious for urothelial carcinoma," and "positive for urothelial carcinoma" were utilized. After initial evaluation, diagnostic criteria of TPS were applied to categorize lesions as either "negative for HGUC," "atypical urothelial cells," "suspicious for HGUC," "positive for HGUC," or "low-grade urothelial neoplasm". Cytologic features including increased nuclear to cytoplasmic $(\mathrm{N}: \mathrm{C})$ ratios, nuclear hyperchromasia, irregular nuclear contours, coarse chromatin distribution, and nuclear pleomorphism as well as the presence of mitotic figures, urothelial cells with atypical degeneration, and bizarre single cells were recorded for each specimen. The frequency of changed diagnoses before and after application of TPS diagnostic criteria, concordance with subsequent surgical resection specimens (which was considered to be the gold standard for each patient), and the presence of distinct cytologic features in patients with subsequent resections were evaluated. Slides from the surgical resections were reviewed by 3 study pathologists (C.T.S., S.L.S. and R.M.) to confirm the pathologic features including the diagnosis, grade, and stage.

Associations between the cytologic diagnoses, features, and resection grade were examined using the $\chi 2$ test or Fisher's exact test, as indicated; a P-value of $<.05$ was considered to be statistically significant.

\section{3 | RESULTS}

Demographic data from the patient cohort is summarized in Table 1. Of the 53 patients, 44 were male (83\%). Patient age ranged from 47-94 years at the time of surgical resection. In total, 13 of the patients had either received either neoadjuvant chemotherapy (8\%) or adjuvant therapy (17\%). Disease progression was noted as clinical recurrence in 20 patients (38\%) and 10 (19\%) patients had definitive metastasis (4 patients with urothelial carcinoma involving 1 metastatic site, 3 patients with 2 metastatic sites, 1 patient with 3, 1 patient with 4 , and 1 patient with 5). The most common metastatic site was lung (6 cases), followed by liver ( 5 cases), bone ( 4 cases), kidney ( 2 cases),

TABLE 1 Demographics for 53 patients

\begin{tabular}{|lll}
\hline Gender $n$ (\%) & Male & $44(83 \%)$ \\
\hline Age (years) & Female & $9(17 \%)$ \\
& Range & $47-94$ \\
& Mean & 69 \\
& Median & 69 \\
\hline Therapy $n$ (\%) & Neoadjuvant chemotherapy & $4(8 \%)$ \\
& Adjuvant therapy & $9(17 \%)$ \\
\hline Previous cystectomy & & $7(13 \%)$ \\
for bladder cancer $n$ (\%) & & $20(38 \%)$ \\
Outcomes $n$ (\%) & Recurrence & $10(19 \%)$ \\
\hline Time to last follow up (days) & Range & $7-3635$ \\
& Metastasis & 1176
\end{tabular}


soft tissue ( 2 cases), axillary lymph nodes ( 2 cases), and prostate (1 case). The time to last clinical follow-up ranged from 7 to 3635 days.

Of the 54 surgical resections evaluated, 23 were from the right (43\%) and 31 were from the left side (57\%). The majority of these resections were diagnosed as HGUC ( $n=41,76 \%$ ), while low-grade urothelial carcinoma (LGUC) was diagnosed in the remaining cases ( $n=13,24 \%$ ). The resection specimen data is summarized in Table 2.

A total of 61 ipsilateral urine specimens collected 5-323 days prior to resection were evaluated before and after application of diagnostic criteria put forth by TPS. Table 3 summarizes cytologic specimen collection data. Application of TPS criteria resulted in a change of diagnosis in 16 (26\%) cases. Of these, 16 cases, 10 (63\%) were diagnostic downgrades while the remaining $6(37 \%)$ resulted in diagnostic upgrades. Of the 6 upgrades, $3(50 \%)$ represented a change from the atypical to suspicious category, 2 (33\%) represented a change from the negative to atypical category, and 1 (17\%) represented a change from suspicious to positive. Of the 10 downgrades, the most common change was from positive to suspicious (6, 60\%). The remaining 4 downgrades were split between downgrades from suspicious to atypical and atypical to negative. Table 4 summarizes the results of applying TPS criteria to the upper tract urine specimens on diagnostic rates of all diagnostic categories.

The frequency of cytologic features associated with subsequent LGUC and HGUC on surgical resection was evaluated. The presence of coarse chromatin distribution among atypical urothelial cells (Figure $1 \mathrm{~A}, \mathrm{C}$ ) and bizarre single cells were seen more commonly in HGUC in comparison to LGUC; these findings were statistically significant. In contrast, nuclear to cytoplasmic ratios, nuclear hyperchromasia, nuclear contour irregularities, pleomorphism, as well as the presence of mitoses and/or atypical degeneration were not statistically significant features in differentiating between LGUCs and HGUCs (Table 5).

The concordance rate between cytologic diagnoses and the subsequent surgical resection was evaluated. The sensitivity of a positive diagnosis in upper urinary tract cytology in the detection of HGUC prior to the application of TPS criteria was $29 \%$ and decreased to $19 \%$ after application of TPS criteria. However, the sensitivities in detecting HGUC are more similar after suspicious and atypical categories were included into the "positive" cohort (sensitivities of $60 \%$ when including the suspicious category into the "positive" cohort before and after application of TPS criteria, sensitivities of $88 \%$ and $85 \%$ after including both suspicious and atypical categories in the "positive cohort" before and after application of TPS criteria, respectively). For LGUC, the sensitivity of upper urinary tract urine cytology is much lower than that for HGUC, which is expected given that the primary focus of urine cytology stated by the TPS is the detection of HGUC. Evaluation of upper urinary tract cytology both before and after

TABLE 2 Surgical resections for upper tract urothelial carcinoma

$\begin{array}{lll}N=54 & & n(\%) \\ \text { Laterality } & \text { Right } & 23(43) \\ & \text { Left } & 31(57) \\ \text { Grade } & \text { Low } & 13(24 \%) \\ & \text { High } & 41(76 \%)\end{array}$

TABLE 3 Cytology specimen summary

\begin{tabular}{lll}
$\begin{array}{c}\text { Total number of upper tract cytology } \\
\text { specimens within 1 year of resection }\end{array}$ & 61 \\
\hline Cytology specimens per resection n (\%) & 1 & $47(87 \%)$ \\
\hline Site of urine cytology collection $n(\%)$ & 2 & $7(13 \%)$ \\
& Renal pelvis & $37(61 \%)$ \\
\hline Time from proximal cytology specimen to & Ureter & $24(39 \%)$ \\
\hline surgical resection (days) & Range & $5-323$ \\
& Mean & 66 \\
& Median & 45
\end{tabular}

application of TPS shows higher sensitivity in the detection of HGUC in comparison to LGUC. The sensitivities of upper urinary tract cytology before and after application of TPS are shown for each diagnostic category in Table 6.

Of the 6 cases that were upgraded after application of TPS, all displayed $\mathrm{N}: \mathrm{C}$ ratios $\geq 0.7$ and nuclear hyperchromasia. The 1 case that was upgraded from suspicious to positive showed all key diagnostic features of HGUC highlighted in TPS criteria ( $\mathrm{N}: \mathrm{C}$ ratio $\geq 0.7$, nuclear hyperchromasia, and coarse chromatin distribution) in cells with relatively small nuclei (Figure 2A,B). Of the 10 cases that were downgraded with application of TPS, 8 cases (80\%) showed N:C ratios $\geq 0.7$, nuclear hyperchromasia, and pleomorphism. In our cohort, 6 of the 10 downgraded cases (60\%) represented changes from positive to suspicious diagnostic categories. In these cases, the cells displayed $\mathrm{N}: \mathrm{C}$ ratios $\geq 0.7$, nuclear hyperchromasia, and coarse chromatin distribution, but lacked markedly irregular nuclear contours (Figure 3A, E) seen in other cases diagnosed as positive for HGUC (Figure 4A,B). All $10(100 \%)$ of the cases that were downgraded were diagnosed as HGUC on resection, compared with 4 of $6(66 \%)$ cases that were upgraded after application of TPS criteria.

\section{4 | DISCUSSION}

Prior to TPS, the institutional diagnostic rate of atypical urine cytology ranged from $2 \%$ to $31 \%$ with $8.3-37.5 \%$ of these cases associated with a subsequent histologic diagnosis of HGUC. ${ }^{9-11}$ Taking note of the inconsistent utilization of the "atypical" category in urine cytology and its variable association with subsequent urothelial carcinoma diagnoses on biopsies/resections, the authors of TPS established the goal of creating diagnostically more reproducible and, hopefully, more clinically meaningful diagnostic categories.

The utilization of TPS has yielded variable results, most of which are favorable, after implementation across institutions. Some institutions have noted that the most significant change seen from pre to post-Paris System evaluation lies in the diagnostic rate of the indeterminate category of "atypical" and, to a lesser degree, the "suspicious" category. Although many institutions have displayed a decreased atypical diagnostic rate after implementing TPS diagnostic criteria, ${ }^{9,12,13}$ few have reported no significant changes in the rate of atypical urine cytology ${ }^{14}$ and rare others have reported an increased atypical rate. ${ }^{15}$ Of note, many of the studies that showed a decrease in diagnostic rates of "atypical" urine cytology also reported an increased association of these "atypical" cases with 
TABLE 4 Cytology diagnoses before and after application of Paris System Criteria

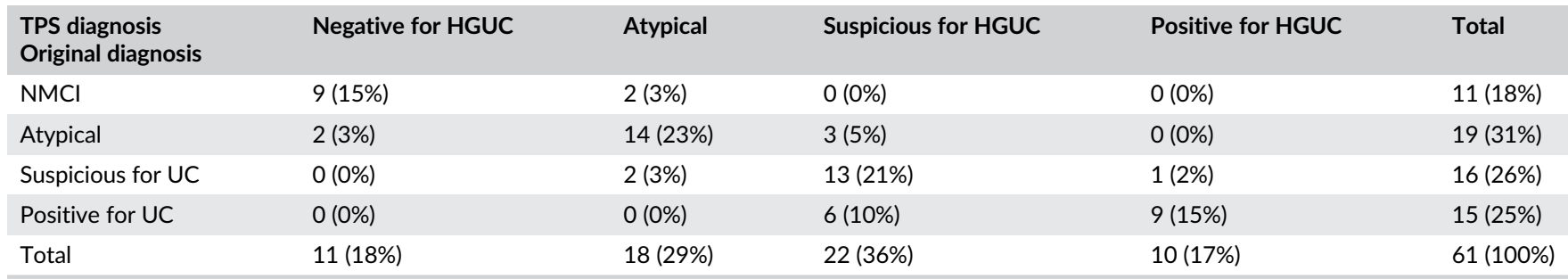

Abbreviations: HGUC, high-grade urothelial carcinoma; $\mathrm{NMCl}$, negative for malignant cells; UC, urothelial carcinoma.

(A)

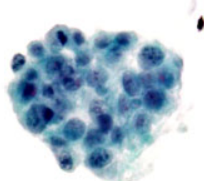

:
(B)

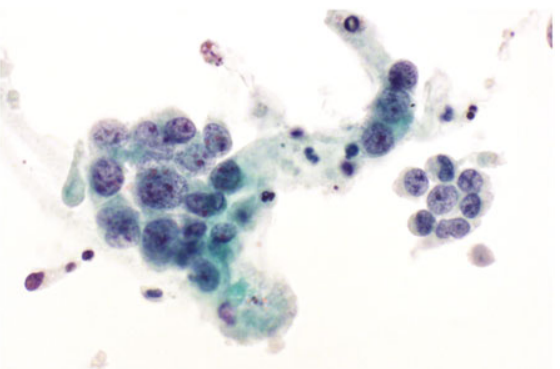

(C)

FIGURE 1 Atypical urothelial cells display coarse chromatin distribution as well as nuclear pleomorphism with cell clusters (ThinPrep, 400X magnification-A, ThinPrep, 600× magnification-B,C) [Color figure can be viewed at wileyonlinelibrary.com]

subsequent histologic diagnoses of HGUC. Additionally, several studies have noted the improvement in correlation of urinary cytology when using TPS classification with subsequent biopsy diagnoses. ${ }^{12,14,16,17}$ However, while many of these studies have focused on the evaluation of urine cytology specimens derived from voided urines or bladder washings evaluating the lower urinary tract, there have few studies with a predominant focus on upper urinary tract cytology.

In our current study focusing on upper urinary tract cytology, the most common cytologic features associated with HGUC on subsequent resections were $\mathrm{N}: \mathrm{C}$ ratios $\geq 0.7$ (88\% of urine cytology cases from patients with subsequent HGUC on resection), hyperchromasia (83\%), coarse chromatin distribution (67\%), and nuclear pleomorphism within cell clusters (65\%). Application of TPS diagnostic criteria resulted in a slightly increased use of the suspicious diagnostic category $(26 \%$ to $36 \%$ ) with no significant change in the diagnostic rate of the atypical diagnostic category. Zheng et al., ${ }^{18}$ also evaluated the effect of utilizing TPS in 125 upper urinary tract urine cytology specimens from 74 patients who had subsequent biopsies or resections within 2 months of upper urinary tract urine collection. In this study, the diagnostic rates in this cohort for non-diagnostic, negative for HGUC, atypical urothelial cells, low-grade urothelial neoplasm, suspicious for HGUC, and positive for HGUC were 1.6\%, 39.2\%, 17.6\%,

TABLE 5 Cytologic features of upper tract urine cytology specimens in HGUC and LGUC

\begin{tabular}{|c|c|c|c|c|c|c|c|c|c|}
\hline & $\begin{array}{l}\text { No. of } \\
\text { atypical cells }\end{array}$ & $\mathrm{N}: \mathrm{C} \geq 0.7$ & $\begin{array}{l}\text { Nuclear } \\
\text { hyperchromasia }\end{array}$ & $\begin{array}{l}\text { Nuclear contour } \\
\text { irregularity }\end{array}$ & $\begin{array}{l}\text { Coarse } \\
\text { chromatin }\end{array}$ & Pleomorphism & Mitoses & $\begin{array}{l}\text { Atypical } \\
\text { degeneration }\end{array}$ & $\begin{array}{l}\text { Bizarre } \\
\text { single cells }\end{array}$ \\
\hline \multirow[t]{3}{*}{$\operatorname{HGUC~}(n=48)$} & $\leq 10: 15$ (31\%) & 42 (88\%) & $40(83 \%)$ & $11(23 \%)$ & $32(67 \%)$ & $31(65 \%)$ & $6(13 \%)$ & $7(15 \%)$ & $18(38 \%)$ \\
\hline & $11-20: 9$ (19\%) & & & & & & & & \\
\hline & $>20: 14$ (50\%) & & & & & & & & \\
\hline \multirow[t]{3}{*}{ LGUC ( $n=13)$} & $\leq 10: 8(62 \%)$ & $10(77 \%)$ & $9(69 \%)$ & $4(31 \%)$ & $1(7 \%)$ & $5(36 \%)$ & $0(\%)$ & $0(0 \%)$ & $1(7 \%)$ \\
\hline & $11-20: 2(15 \%)$ & & & & & & & & \\
\hline & $>20: 3(23 \%)$ & & & & & & & & \\
\hline$P$ & $\mathrm{~N} / \mathrm{A}$ & 0.340 & .257 & .560 & $<.001$ & .089 & .326 & .328 & .040 \\
\hline
\end{tabular}


TABLE 6 Sensitivity of upper tract urine cytology in detecting HGUC and LGUC

\begin{tabular}{llll} 
& HGUC & LGUC & LG and HG \\
\hline Original diagnosis & & & \\
Positive & 0.29 & 0.08 & 0.25 \\
\hline Positive + suspicious & 0.60 & 0.15 & 0.59 \\
\hline Positive + suspicious + atypical & 0.88 & 0.62 & 0.82 \\
\hline TPS diagnosis & & & \\
\hline Positive & & & \\
\hline Positive + suspicious & 0.19 & 0.08 & 0.16 \\
\hline Positive + suspicious + atypical & 0.60 & 0.21 & 0.52 \\
\hline
\end{tabular}

Abbrviations: HGUC, high-grade urothelial carcinoma; LGUC, low-grade urothelial carcinoma.

$8.8 \%, 16.8 \%$, and $16 \%$, respectively. However, the study did not evaluate the diagnostic rates of these categories prior to implementation of TPS diagnostic criteria. Zheng et al., also reported higher sensitivity for the detection of HGUC when cytology was diagnosed as positive for HGUC than our study (45\% vs $19 \%$ ). However, similar to our study, the sensitivity significantly increased when both suspicious and positive were considered "positive" (78.6\% vs $60 \%$,) and even more so when the atypical diagnostic category is also considered as part of the positive cohort ( $92.9 \%$ vs $85 \%)$. In a more recent study by Mclntire et al., ${ }^{19}$ implementation of TPS criteria also resulted in an improved correlation of upper urinary tract cytology specimens with subsequent biopsies (from $34 \%$ to $63 \%$ ) and a decreased use in of the atypical diagnostic category (from $29 \%$ to 13\%). However, in contrast to our current study, the pre-TPS original diagnoses were gathered from the prior pathology reports and were not re-reviewed and distinct cytologic features of the urine specimens were not evaluated.

As noted previously in our results, there was a diagnostic category change after application of TPS criteria in 16 (26\%) cases, 10 of which represent diagnostic downgrades while the others represent diagnostic upgrades. The most common upgrade was seen in cases originally diagnosed as atypical and then called suspicious for HGUC after TPS criteria were applied (50\%, 3 of 6 upgrades) while the most common downgrade was seen in cases originally diagnosed as positive for urothelial carcinoma and then called suspicious for HGUC after strict TPS criteria were applied (60\%, 6 of 10 downgrades). Upon rereview of the cases resulting in diagnostic changes, we found that the 1 case resulting in an upgrade from suspicious for urothelial carcinoma to positive for HGUC after application of TPS criteria displayed
(A)

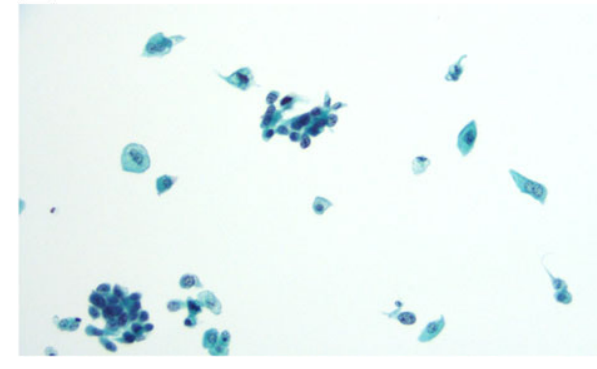

(B)

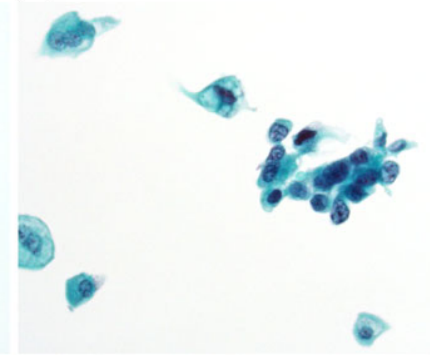

FIGURE 2 Features of case associated with diagnostic upgrade from suspicious to positive after application of TPS criteria. Atypical urothelial cells display $\mathrm{N}: \mathrm{C}$ ratios $>0.7$, nuclear hyperchromasia, coarse chromatin distribution, and nuclear contour irregularity. However, the overall nuclear size is not markedly enlarged compared with background benign urothelial cells (ThinPrep, 200x magnification-A, 400× magnification-B) [Color figure can be viewed at wileyonlinelibrary.com]

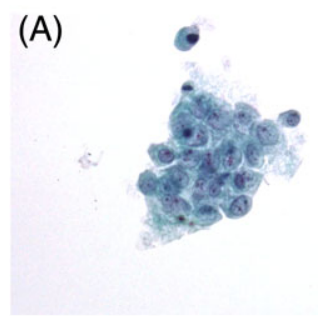

(B)
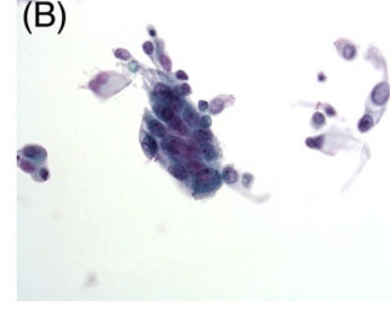

(C)

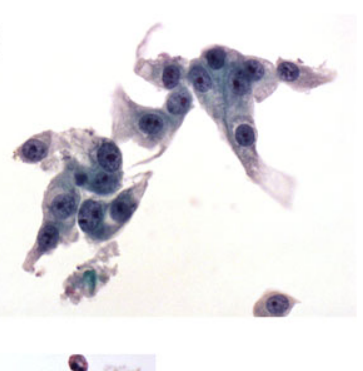

(D)

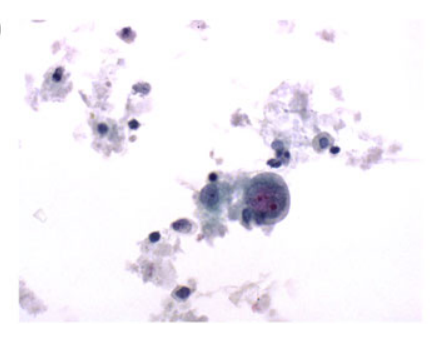

(E)

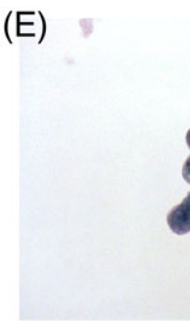

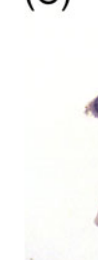

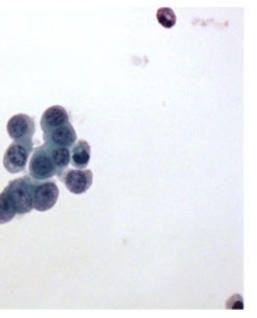

FIGURE 3 Features of cases associated with diagnostic downgrade from positive to suspicious after application of TPS criteria. Atypical urothelial cells display increased $\mathrm{N}: \mathrm{C}$ ratios, nuclear hyperchromasia, prominent nucleoli, and coarse chromatin distribution, but lack marked nuclear contour irregularity (ThinPrep, 400x magnification-A,B, 600x magnification-C-E) [Color figure can be viewed at wileyonlinelibrary.com] 


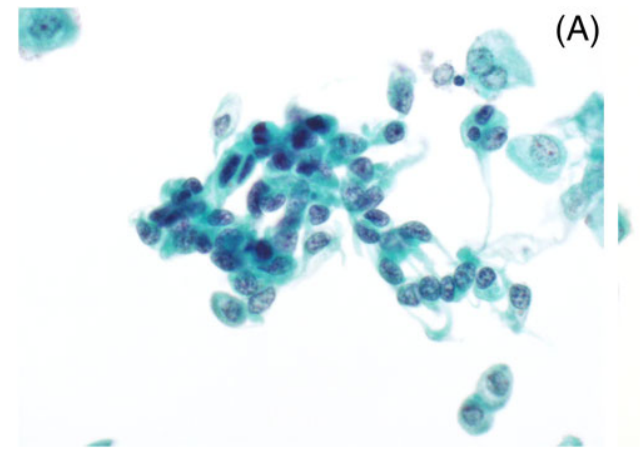

(B)

FIGURE 4 Atypical urothelial cells with irregular nuclear contours in cases diagnosed as positive for HGUC after application of TPS criteria (ThinPrep, 600x magnification-A,B) [Color figure can be viewed at wileyonlinelibrary.com]

atypical urothelial cells with $\mathrm{N}: \mathrm{C}$ ratios $\geq 0.7$, coarse chromatin distribution, nuclear hyperchromasia, and nuclear contour irregularity in cells; however, these features were seen in urothelial cells with relatively small nuclei compared with surrounding benign urothelial cells (Figure 2A,B). Additionally, the most consistent feature associated with a diagnostic downgrade from positive for urothelial carcinoma to suspicious for HGUC was the lack of marked nuclear contour irregularity in atypical urothelial cells (Figures $3 \mathrm{~A}, \mathrm{E}$ ), one of the key Paris System diagnostic features of malignant urothelial cells. This finding highlights how strict application of TPS criteria for upper urinary tract cytology specimens can decrease the frequency of positive diagnoses.

Our findings may indicate that different morphologic features, particularly markedly irregular nuclear contours, may have different predictive values for HGUC in upper urinary tract urine cytology specimens compared with those from the lower urinary tract. However, there are limitations to this current study, including the retrospective nature of patient selection. This could provide a source of selection bias, as slides were reviewed knowing that subsequent resection specimens had either LGUC or HGUC. As such, mild cytologic features may be more carefully evaluated. Additionally, the cytology specimens were only re-reviewed by 1 cytopathologist during the study, which does not allow for a consensus opinion. Nonetheless, if similar findings are validated by other independent studies, future iterations of TPS may consider revision of diagnostic criteria, particularly for upper tract urine specimens.

Suh et al., evaluated 142 urinary tract washing specimens derived from the urinary bladder, ureter, and renal pelvis for cytologic features (including anisonucleosis, India ink nuclei, apoptotic bodies, increased $\mathrm{N}: \mathrm{C}$ ratios, irregular nuclear membranes, coarse chromatin, hyperchromasia, pleomorphism, tumor diathesis) associated with HGUC. After regression analyses, anisonucleosis and India ink nuclei were reported to be independent predictors for HGUC. The authors then applied a modified TPS to include these 2 features, which resulted in an increased sensitivity in the detection of HGUC without a significant loss in specificity. Another study of 112 urinary tract cytology specimens from patients with a subsequent diagnosis of HGUC on tissue biopsy by Cowan et al. ${ }^{20}$ noted that strict application of TPS criteria (requiring the presence of a $\mathrm{N}: \mathrm{C}$ ratio $\geq 0.7$, nuclear hyperchromasia, markedly irregular nuclear contours, and coarse chromatin distribution as well as a minimum quantity of atypical urothelial cells with the aforementioned features) could potentially restrict a diagnosis of
HGUC. Although the study showed that $\mathrm{N}: \mathrm{C}$ ratio $\geq 0.7$ was the most stringent TPS diagnostic criteria (78\%), it also noted that smooth nuclear contours in HGUC cells could be a potential pitfall in the evaluation of urine cytology specimens, as markedly irregular nuclear contours were identified in $83 \%$ of specimens. However, both of these studies did not restrict their evaluation to upper urinary tract specimens or substratify their findings between lower and upper tract specimens. Nonetheless, the findings of these studies also suggest that perhaps more fluid application of TPS diagnostic criteria, in contrast to strict adherence to all its diagnostic criteria, as well as potential revisions of diagnostic criteria in future iterations of TPS may increase the diagnostic accuracy of urine cytology.

A potential revision of diagnostic criteria for urothelial carcinoma in both upper and lower urinary tract may affect not only diagnostic accuracy but also reproducibility. More studies are needed to establish the effect TPS has had on diagnostic accuracy and inter-observer variability, as well as to further evaluate key morphologic features, such as the presence or absence of nuclear contour irregularities, that can affect diagnostic accuracy in upper urinary tract cytology specimens compared with lower urinary tract specimens. Modification of diagnostic criteria based on the source of urinary cytology specimens may enhance the utility of urine cytology and eventually help develop clinically meaningful management guidelines for patient care.

\section{Precis}

The Paris System introduced diagnostic criteria for urine cytology to improve reproducibility among pathologists. However, modifications to diagnostic criteria, particularly in the upper urinary tract may enhance its diagnostic accuracy.

\section{ORCID}

Madelyn Lew (D) https://orcid.org/0000-0002-6279-0330

\section{REFERENCES}

1. Ploeg M, Aben KK, Kiemeney LA. The present and future burden of urinary bladder cancer in the world. World J Urol. 2009;27(3):289-293.

2. Fiuk JV, Schwartz BF. Upper tract urothelial carcinoma: paradigm shift towards nephron sparing management. World J Nephrol. 2016;5(2): 158-165. 
3. Margulis V, Shariat SF, Matin SF, et al. Outcomes of radical nephroureterectomy: a series from the upper tract Urothelial carcinoma collaboration. Cancer. 2009;115:1224-1233.

4. Siegel RL, Miller KD, Jemal A. Cancer statistics, 2018. CA Cancer J Clin. 2018;68(1):7-30.

5. Koss LG, Deitch D, Ramanathan R, Sherman AB. Diagnostic value of cytology of voided urine. Acta Cytol. 1985;29(5):810-816.

6. Zhang ML, Rosenthal DL, VandenBussche CJ. Upper urinary tract washings outperform voided urine specimens to detect upper tract high-grade urothelial carcinoma. Diagn Cytopathol. 2017;45(8): 700-704.

7. Bagley DH, Huffman JL, Lyon ES. Flexible ureteropyeloscopy: diagnosis and treatment in the upper urinary tract. J Urol. 1987;138:280-285.

8. Owens $\mathrm{CL}$, VandenBussche $\mathrm{CJ}$, Burroughs FH, Rosenthal DL. A review of reporting systems and terminology for urine cytology. Cancer Cytopathol. 2013;121(1):9-14.

9. Wang Y, Auger M, Kaner Y, Cagler D, Brimo F. Implementing The Paris System for Reporting Urinary Cytology results in a decrease in the rate of the "atypical" category and an increase in its prediction of subsequent high-grade urothelial carcinoma. Cancer Cytopathol. 2018; 126(3):207-214.

10. Brimo F, Vollmer RT, Case B, Aprikian A, Kassouf W, Auger M. Accuracy of urine cytology and the significance of an atypical category. Am J Clin Pathol. 2009;132:785-793.

11. Mokhta GA, Al-Dousar M, Al-Ghamedi D. Diagnostic significance of atypical category in the voided urine samples: a retrospective study in a tertiary care center. Urol Ann. 2010;2:100-106.

12. Hassan M, Solanki S, Kassouf W, et al. Impact of implementing the Paris System for Reporting Urine Cytology in the performance of urine cytology: a correlative study of 124 cases. Am J Clin Pathol. 2016;145: 384-390.

13. Suh J, Go H, Sung C, et al. Modification of the Paris System for urinary tract washing specimens using diagnostic cytologic features. Cytopathology. 2017;28:516-523.
14. Meilleroux J, Daniel G, Aziza J, et al. One year of experience using the Paris System for Reporting Urinary Cytology. Cancer Cytopathol. 2018;126:430-436.

15. Granados R, Duarte JA, Corrales T, Camarmo E, Bajo E. Applying the Paris System for Reporting Urine Cytology increases the rate of atypical urothelial cells in benign cases: a need for patient management recommendations. Acta Cytol. 2017;61:71-76.

16. Bertsch EC, Siddiqui MT, Ellis CT. The Paris system for reporting urinary cytology improves correlation with surgical pathology diagnosis of the lower urinary tract. Diagn Cytopathol. 2018;46(3):221-227.

17. Cowan ML, VandenBussche CJ. The Paris System for Reporting Urinary Cytology: early review of the literature reveals successes and rare shortcomings. J Am Soc Cytopathol. 2018;7(4):184-194.

18. Zheng X, Si Q, Du D, et al. The Paris System for urine cytology in upper tract urothelial specimens: a comparative analysis with biopsy and surgical resection. Cytopathology. 2018;29(2):184-188.

19. McIntire PJ, Snow JT, Robinson BD, et al. Improved correlation of urinary cytology specimens using The Paris System in biopsy-proven upper tract urothelial carcinomas. Cancer Cytopathol. 2018;126(7): 498-504.

20. Cowan ML, Rosenthal DL, VandenBussche CJ. Improved risk stratification for patients with high-grade urothelial carcinoma following application the Paris System for Reporting Urinary Cytology. Cancer Cytopathol. 2017;125(6):427-434.

How to cite this article: Simon CT, Skala SL, Magers MJ, et al. The utility of upper urinary tract urine cytology before and after application of the Paris system. Diagnostic Cytopathology. 2019;47:421-427. https://doi.org/10.1002/dc.24127 